\title{
Treatment algorithms for hepatitis B and C
}

\author{
E R Schiff
}

\begin{abstract}
While several predictors of an early and sustained response can be identified in the treatment of chronic hepatitis $B$, no consistently demonstrable predictors of response exist for chronic hepatitis C. As such, guidelines for the treatment of chronic hepatitis $C$ are more complex than those for hepatitis B. The treatment options for chronic hepatitis $B$ and $C$ are outlined and there is speculation on the clinical challenges of the future.

(Gut 1993; supplement: S148-S149)
\end{abstract}

Interferons have emerged as the group of antiviral agents with proved efficacy in the treatment of chronic viral hepatitis $\mathrm{B}$ and $\mathrm{C}$ (HBV and HCV, respectively). For optimum effect, treatment has to be tailored to the patient's needs, taking into consideration the patient's age, the stage of their disease, their motivation, and toleration of the drug.

We are now approaching the stage where the monitoring of treatment by HBV-DNA and HCV-RNA determination should provide a more rational treatment strategy for chronic hepatitis B and C, respectively. Predictors of a favourable response to alpha interferon in hepatitis $B$ and $C$ are shown in the Table.
TABLE Predictors of a favourable response to alpha interferon treatment in hepatitis $B$ and $C$

\begin{tabular}{ll}
\hline Hepatitis $B$ & Hepatitis $C$ \\
\hline HBV-DNA $<200 \mathrm{pg} / \mathrm{ml}$ & \\
ALT $>100 \mathrm{IU} / \mathrm{l}$ & \\
Female sex & $\begin{array}{l}\text { No consistently demonstrable } \\
\text { Short duration of infection }\end{array}$ \\
Anti-HIV negativity & \\
Heterosexuality & \\
Non-Asian origin & \\
\hline
\end{tabular}

*Preliminary evidence suggests disappearance of HCV-RNA may be the best predictor of an early and sustained response. ALT=alanine aminotransferase.

\section{Chronic hepatitis B}

In most cases, chronic viral hepatitis $B$ is treated with 5 million units (MU) interferon daily or $10 \mathrm{MU}$ three times weekly for four months. If treatment leads to loss of HBVDNA and a return to normal of serum transaminase activities, there is probably no need to give more than four months of therapy. The reactivation rate is low and, with time, these individuals are likely to go on to lose surface antigen. Studies have shown that treatment with interferon alfa-2b leads to the disappearance of HBV-DNA and the seroconversion of e antigen in about $40 \%$ of patients with chronic viral hepatitis $B$.

If, at four months, the HBV-DNA value is still falling, treatment should be continued
University of Miami School of Medicine and Veterans

Administration

Medical Center,

Miami, Florida, USA

E R Schiff

Correspondence to:

Professor E R Schiff,

VA Medical Center,

1201 Northwest 16 th Street,

Room D1011 (111H),

Miami, Florida 33125, USA.

\section{Start 3 MU IFN TIW}

3 months

\section{6} months

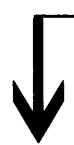


until this becomes negative. Problems arise when patients do not respond at all to an initial course of interferon. In these patients it is reasonable to try another antiviral or adjunctive therapy.

\section{Chronic hepatitis C}

Guidelines for the treatment of chronic hepatitis $\mathrm{C}$ are more complex than those for $\mathrm{B}$. A suggested algorithm for the treatment of chronic hepatitis $\mathrm{C}$ is shown in the figure. Treatment should begin with $3 \mathrm{MU}$ interferon three times weekly for three months, and should continue until the HCV-RNA value is negative. The dose should then be decreased to $1 \mathrm{MU}$ three times weekly and treatment continued for one year. If patients relapse when treatment is withdrawn, retreating with the initial regimen is recommended, providing there are no contraindications. In the case of non-responders, where the HCV-RNA value actually increases, the dose of interferon should be increased to $5 \mathrm{MU}$ three times weekly. If, after three months, the HCV-RNA is still showing no signs of a reduction, treatment with interferon should be stopped and another agent considered. Ribavirin and thymosin may have a place in treating chronic hepatitis, either alone or in combination with interferon. Although ribavirin has the advantage of oral administration, studies have not shown an impressive rate of complete response.

\section{Future challenges}

Although interferons are proved to be effective in the treatment of chronic viral hepatitis B and $C$, there are still some challenges ahead. Certain situations remain quite difficult, for example the post liver transplant patient who almost inevitably becomes re-infected both in the case of chronic $B$ and chronic $C$ hepatitis. Should we be treating more of these patients before transplantation? Can we use a regimen after transplantation without bringing about rejection? Immunosuppressed people, for example HIV positive patients, are also difficult to treat, as are patients with autoimmune disease. Finally, one of the biggest challenges facing us in the world is the perinatal transmission of viral hepatitis. The vast majority of people who are carriers of $\mathrm{HBV}$ were infected in this way, and as a group are relatively refractory to treatment with interferon. 